\title{
Oncological Outcome of Primary versus Secondary Muscle-Invasive Bladder Cancer Is Comparable after Radical Cystectomy
}

\author{
A. Aziz ${ }^{a} \quad$ M. Gierth ${ }^{a} \quad$ H.M. Fritsche ${ }^{a} \quad$ M. May ${ }^{b} \quad$ W. Otto ${ }^{a} \quad$ S. Denzinger ${ }^{a}$ \\ W.F. Wieland ${ }^{a} \quad$ A. Merseburger ${ }^{c} \quad$ H. Riedmiller ${ }^{d} \quad$ A. Kocot ${ }^{d} \quad$ M. Burger $^{d}$ \\ ${ }^{a}$ Department of Urology, Caritas-St. Josef Medical Centre, University of Regensburg, Regensburg, ${ }^{b}$ Department of \\ Urology, St. Elisabeth Klinikum Straubing, Straubing, ' Department of Urology and Urooncology, Medical School \\ of Hanover, Hanover, and d Department of Urology and Pediatric Urology, Julius-Maximilians University Medical \\ Centre, Würzburg, Germany
}

\section{Key Words}

Bladder cancer - Risk stratification - EORTC risk tables .

Prognosis · Progression · Radical cystectomy

\begin{abstract}
Background: High-risk non-muscle-invasive bladder cancer (NMIBC) progressing to muscle-invasive bladder cancer (MIBC) is associated with adverse tumour biology. It is unclear, however, whether outcome of NMIBC progressing to $M I B C$ is adverse compared to primary MIBC and whether NMIBC of higher risk of progression to MIBC is adverse compared to NMIBC of lower risk. Objective: Our objective was to assess cancer-specific survival (CSS) following radical cystectomy (RC) for primary MIBC and for NMIBC progressing to MIBC in dependence of EORTC risk score. Materials and Methods: Clinical and histopathological characteristics and CSS of 150 patients were assessed. Secondary MIBCs were stratified by EORTC risk score at the last transurethral resection of bladder tumour for NMIBC. Results: CSS did not differ significantly between primary and secondary MIBC $(p=$ 0.521). Secondary MIBC with high EORTC score had significantly shorter CSS compared to secondary MIBC with intermediate EORTC score $(p=0.029)$. In multivariable analysis,
\end{abstract}

pathological tumour stage $(\mathrm{HR}=3.77 ; \mathrm{p}=0.020)$ and lymph node stage ( $H R=2.34 ; p=0.022$ ) were significantly correlated with CSS. Conclusion: While the outcome of secondary MIBC is not generally adverse compared to primary MIBC, the EORTC risk score not only reflects high risk of progression of NMIBC to MIBC, but also worse outcome following RC for secondary MIBC. Timely RC should thus be debated in highrisk NMIBC.

Copyright $\odot 2013$ S. Karger AG, Basel

\section{Introduction}

Bladder cancer is the fourth most common cancer in men with severe impact on general health; it is generally divided into non-muscle-invasive bladder cancer (NMIBC) and muscle-invasive bladder cancer (MIBC) [1]. Current guidelines recommend radical cystectomy (RC) with bilateral pelvic lymphadenectomy in MIBC and in NMIBC with high risk of progression [2,3]. Risk of progression to MIBC is usually determined by the EORTC risk score combining various clinicopathological parameters [2-4]. Risk of cancer-specific mortality following RC for MIBC is usually determined by pathologi-

\section{KARGER}

E-Mail karger@karger.com

www.karger.com/uin
(C) 2013 S. Karger AG, Basel

0042-1138/13/0911-0097\$38.00/0
Atiqullah Aziz, MD

Department of Urology, Caritas-St. Josef Medical Centre

University of Regensburg, Landshuter Strasse 65

DE-93053 Regensburg (Germany)

E-Mail atiqullah.aziz@klinikum.uni-regensburg.de 
cal tumour stage and lymph node status. Since cancerspecific survival (CSS) rates vary widely, other prognostic factors have been evaluated $[5,6]$.

Progression of NMIBC to secondary MIBC, i.e. subsequent to preceding NMIBC as opposed to primary MIBC, could be a potential prognosticator conveying favourable outcome due to the initial non-muscle-invasive character of the bladder cancer or likewise a potential prognosticator conveying adverse outcome due to its progressive tumour biology.

The EORTC risk score predicting progression of NMIBC to MIBC could also be a potential prognosticator conveying adverse outcome by again reflecting progressive tumour biology. Should this scenario be true, debate of timely RC should be emphasized in the management of high-risk NMIBC and such debate should be based on EORTC risk scores. No conclusive data are available to date.

Currently, indications for RC for bladder cancer stages $<$ pT2 are an issue of debate. While long-term bladder preservation is pursued for quality of life reasons, a tendency to advocate RC in case of BCG-refractory and initially recurrent $\mathrm{T} 1$ tumour stage is notable in the recent literature, since favourable long-term outcome has been suggested for timely radical treatment [7-11]. Due to prognostic uncertainty however the clinical management of high-risk NMIBC is demanding and any further prognosticator valuable. We presently analyse CSS in a current series of RC for primary compared to secondary $\mathrm{MIBC}$ to assess prognostic differences and analyse CSS in relation to EORTC risk score at the last transurethral resection of bladder tumour (TURBT) for NMIBC. No such data have been reported to date.

\section{Materials and Methods}

\section{Patient Selection}

Clinical and histopathological data of consecutive patients undergoing RC for clinically localized MIBC between 2004 and 2010 at one tertiary urological centre without neoadjuvant chemotherapy were collected. Figure 1 shows the stratification of the patients into different risk groups. Patients were subdivided into two groups: patients with primary MIBC (group 1) and patients with secondary MIBC (group 2). In order to preserve homogenous patient groups we excluded patients with secondary MIBC, if no intravesical BCG therapy had been performed $(n=11)$ or no re-resection at the time of T1 stage and/or CIS had been performed $(n=3)$. In secondary MIBC, EORTC risk scores at the time of the last TURBT for NMIBC were assessed, resulting in a score from 0 to 23 [4]: (a) number of tumours (single: 0, multiple: 3 ); (b) tumour size ( $<3 \mathrm{~cm}: 0, \geq 3 \mathrm{~cm}: 3$ ); (c) prior recurrence rate (primary: 0 , recurrent: 2); (d) T category (cTa: 0, cT1: 4); (e) presence of con-

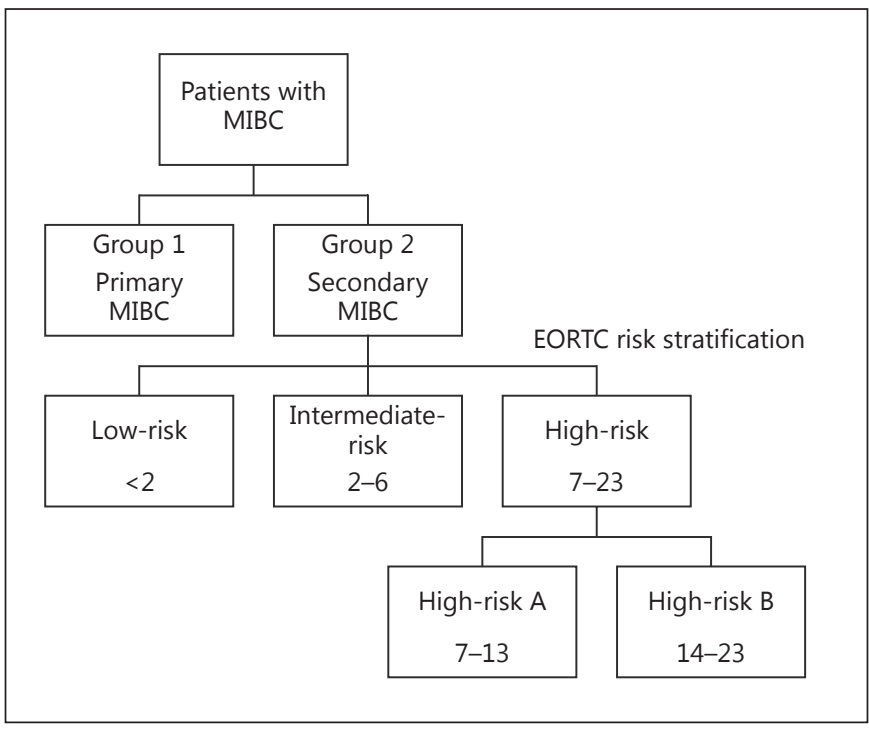

Fig. 1. Risk stratification of the patients according to EORTC.

comitant CIS (no: 0, yes: 6); (f) grade (G1-2: 0, G3: 5). Subsequently, we devised different risk groups, as reported by Sylvester et al. [3]. Due to the frequent occurrence of high-risk patients we subdivided the high-risk group: (a) low-risk group: score $<2$; (b) intermediate-risk group: score 2-6; (c) high-risk group A: score 7-13; (d) high-risk group B: score 14-23. Time from diagnosis of MIBC to RC and from initial TURBT for NMIBC to RC was dichotomised at 90 days in accordance with previous reports [12-14].

\section{Pathological Evaluation}

Surgical specimens were processed according to standard institutional protocols. Tumour grading and staging was performed by genitourinary pathologists according to the 1973 World Health Organization (WHO) grading system and the American Joint Committee on Cancer (AJCC)-Union Internationale Contre le Cancer tumour, nodes, metastasis (TNM) classification, respectively $[15,16]$.

\section{Follow-Up Regimen}

Patients were followed according to institutional guidelines effective at that time largely reflecting current guidelines [2]. For the first and from the second through the fifth year after RC, follow-up visits were scheduled every 3 and 6 months, respectively, and annually thereafter with routine laboratory, ultrasound, urinary cytology, chest radiography, radiographic evaluation of the upper urinary tract and cystoscopic evaluation of the neobladder. Bone scan, computed tomography and/or magnetic resonance imagings were performed when clinically indicated. Cause of death was determined by the treating physician and death certificates.

\section{Statistical Analysis}

The hypothesis of normality for all continuous variables was tested by the Shapiro-Wilk normality test. Means and standard deviations were displayed for the continuous stable distributed variables. Medians with interquartile ranges (IQRs) were used to 
Table 1. Clinical and histopathological characteristics of primary and secondary MIBC

\begin{tabular}{|c|c|c|c|c|}
\hline Criteria & $\begin{array}{l}\text { Entire cohort } \\
(n=150)\end{array}$ & $\begin{array}{l}\text { Primary MIBC } \\
(\mathrm{n}=125)\end{array}$ & $\begin{array}{l}\text { Secondary MIBC } \\
(\mathrm{n}=25)\end{array}$ & $\mathrm{p}$ value \\
\hline Median age (IQR), years & $70(64-76)$ & $69(63-75.2)$ & $71(66.5-77.5)$ & 0.208 \\
\hline Male gender & $121(80.7 \%)$ & $97(77.6 \%)$ & $24(96.0 \%)$ & 0.048 \\
\hline \multicolumn{5}{|l|}{ cT stage, first TURBT } \\
\hline$<\mathrm{cT} 1$ & $11(7.3 \%)$ & 0 & $11(44.0 \%)$ & \multirow[t]{2}{*}{$<0.001$} \\
\hline cT3/4 & $4(2.7 \%)$ & $4(3.2 \%)$ & 0 & \\
\hline Number of TURBT, median (IQR) & $1(1-1)$ & $1(1-1)$ & $3(2-4.5)$ & $<0.001$ \\
\hline Median interval between first TURBT and RC, days (IQR) & $40(25-82)$ & $33.5(23.7-47.5)$ & $539(271.5-1,992.5)$ & $<0.001$ \\
\hline \multicolumn{4}{|l|}{ pT stage, RC } & 0.371 \\
\hline$<\mathrm{pT} 3$ & $57(38.0 \%)$ & $49(39.2 \%)$ & $8(32.0 \%)$ & 0.652 \\
\hline Lymphovascular invasion, RC & $85(56.7 \%)$ & $72(57.6 \%)$ & $13(52.0 \%)$ & 0.662 \\
\hline Median number of lymph nodes removed (IQR) & $18(14-23)$ & $18(13-23)$ & $18(14-23.5)$ & 0.753 \\
\hline Adjuvant chemotherapy & $35(23.6 \%)$ & $29(23.4 \%)$ & $6(25.0 \%)$ & 1.000 \\
\hline
\end{tabular}

present continuous not regularly distributed variables. Student's t test was applied for variables with regular and Wilcoxon rank sum (Mann-Whitney) for variables with irregular distribution, respectively. Categorical variables were compared by the $\chi^{2}$ test. CSS was assessed by the Kaplan-Meier method from date of RC and impact of variables analysed by log-rank test. Impact of variables on CSS was assessed by uni- and multivariable Cox proportional Hazards regression models with backward stepwise elimination of criteria lacking significance. Subjects whose cause of death was not due to MIBC were censored at the time of death for CSS calculation. The statistical significance level was set at $\mathrm{p}<0.05$. All reported $\mathrm{p}$ values were two-sided. Statistical analysis was performed using SPSS ${ }^{\circledR}$ v19.0 (SPSS Inc., Chicago, Ill., USA).

\section{Results}

\section{Clinical and Histopathological Outcome}

Out of 150 patients, 125 (83.3\%) underwent RC for primary MIBC and 25 patients (16.6\%) for secondary MIBC. Clinical and histopathological characteristics are given in table 1 . Mean age did not differ between primary and secondary MIBC (69; IQR 63-75.2 vs. 71; IQR 66.5$77.5 ; \mathrm{p}=0.208)$. Male patients were predominant in both populations (77.6 vs. $96 \%$; $\mathrm{p}=0.048$ ). In secondary MIBC the median number of TURBT was 3 (IQR 2-4.5) and the median time from initial TURBT to RC was 539 days
(IQR 271.5-1,992.5). No differences in the rates of delayed RC ( $>90$ days after diagnosis of muscle invasion; $\mathrm{p}=0.371)$, locally advanced tumour stages (pT3/4; $\mathrm{p}=$ $0.652)$, concomitant CIS ( $\mathrm{p}=0.667)$, lymph node metastasis $(\mathrm{p}=0.824)$, lymphovascular invasion $(\mathrm{p}=0.662)$, median number of lymph nodes removed $(p=0.753)$ and patients undergoing adjuvant chemotherapy $(p=1.000)$ were noted between primary and secondary MIBC. In secondary MIBC EORTC risk score of progression at the last TURBT for NMIBC was intermediate-risk in 6, highrisk $A$ in 9 and high-risk $B$ in 10 patients.

\section{Oncological Outcome}

The median follow-up was 46 months (IQR 31-62). In the entire population, CSS after 1, 3 and 5 years were 83 , 67 and 59\%, respectively. Pathological tumour stage $\mathrm{pT} 3 / 4(\mathrm{HR}=3.77 ; \mathrm{p}=0.020)$ and lymph node metastasis $(\mathrm{HR}=2.34 ; \mathrm{p}=0.022)$ were independently associated with CSS in multivariate analysis (table 2). CSS did not differ significantly between primary and secondary MIBC $(\mathrm{p}=0.521$; fig. 2). Patients with secondary MIBC classified as high-risk A of progression at the last TURBT for NMIBC (14-23) showed significantly worse CSS compared to intermediate-risk or high-risk B patients $(\mathrm{p}=$ 0.029 and $\mathrm{p}=0.033$; fig. 3 ). 
Table 2. Uni- and multivariable Cox regression analysis of CSS

\begin{tabular}{|c|c|c|c|c|}
\hline Criteria & $\begin{array}{l}\text { Univariable analysis, } \\
\text { HR (95\% CI) }\end{array}$ & $\mathrm{p}$ value & $\begin{array}{l}\text { Multivariable analysis, } \\
\text { HR ( } 95 \% \text { CI), backward } \\
\text { stepwise elimination }\end{array}$ & $\mathrm{p}$ value \\
\hline Female gender (referent male) & $1.42(0.68-2.94)$ & 0.351 & & n.s. \\
\hline Interval between first TURBT and RC, cont. & $1.00(1.00-1.00)$ & 0.984 & & n.s. \\
\hline pT3/4 stage, $\mathrm{RC}($ referent $\leq \mathrm{pT} 2)$ & $8.95(3.19-25.05)$ & $<0.001$ & $3.77(1.24-11.47)$ & 0.020 \\
\hline $\mathrm{pN}+$ stage, $\mathrm{RC}$ (referent $\mathrm{pN} 0)$ & $5.05(2.68-9.51)$ & $<0.001$ & $2.34(1.13-4.84)$ & 0.022 \\
\hline Grade 3, RC (referent < grade 3 ) & $3.94(0.54-28.56)$ & 0.175 & & n.s. \\
\hline Concomitant CIS, RC (referent absent CIS) & $0.95(0.53-1.70)$ & 0.858 & & n.s. \\
\hline Lymphovascular invasion, RC (referent absent LVI) & $5.66(2.53-12.68)$ & $<0.001$ & & n.s. \\
\hline Number of lymph nodes removed, cont. & $1.00(0.97-1.03)$ & 0.988 & & n.s. \\
\hline
\end{tabular}

\section{Discussion}

Does Outcome of Primary and Secondary MIBC Differ?

No definitely conclusive data on survival rates after RC for secondary MIBC subsequent to preceding NMIBC compared to primary MIBC have been reported to date and the present literature is arbitrary. In 2002, Yiou et al. [17] compared 43 and 12 patients with primary and secondary MIBC, respectively, reporting no difference in survival. May et al. [18] compared 132 and 57 patients with primary and secondary MIBC, respectively, again finding no difference in outcome or any other clinicopathological parameter. While May at al.'s series was larger than the present one, it was assessed in retrospect from various institutions while the present series was assessed prospectively with homogeneous treatment pattern due to institutional guidelines of one centre. In analogy to the present analyses, those series found no differences in outcome between primary versus secondary $\operatorname{MIBC}[17,18]$. A more recent retrospective series by de Vries et al. [19] assessing 134 and 54 patients with primary and secondary MIBC, respectively, found comparable survival rates. The most recent series by Kotb et al. [20] analysed 1,150 patients from the Canadian Bladder Cancer Network containing $32 \%$ of patients with secondary MIBC. In this series, outcome of secondary MIBC was advantageous, as CSS at 5 years was $70 \%$ compared to $60 \%$ of primary MIBC. While age at MIBC was comparable, many other factors were adverse in primary MIBC, e.g. presence of preoperative hydronephrosis ( 20.8 vs. $32.6 \% ; \mathrm{p}=0.0007$ ), rate of higher pathological stage (T3/T4; 36.3 vs. $58.0 \%$; $\mathrm{p}=0.0001)$, positive lymph nodes (20.1 vs. $28.8 \% ; \mathrm{p}=$ 0.002 ) and rates of adjuvant chemotherapy (15.5 vs. $23.3 \% ; \mathrm{p}=0.002$ ). The authors concluded that secondary MIBC may have better clinical and pathological outcome. In contrast to the present series no details on NMIBC prior to MIBC were available and according to the authors different approaches toward early cystectomy in each centre might have biased the results [20].

In accordance with the former series and in contrast to the most recent analysis our data again do not suggest generally adverse outcome of either variation. No other factor biasing CSS was noted in the present series. The ratio of patients with secondary MIBC of all patients undergoing $\mathrm{RC}$ for MIBC in the present series was $25 \%$ and thus within the range of previous series (21.4 to $29.4 \%$ ) [18-22]. No differences in e.g. tumours stage, lymph node status or use of adjuvant chemotherapy were noted. Thus the present homogeneous and well-controlled data, also including detailed information of NMIBC preceding MIBC, suggest comparable outcome for primary and secondary MIBC.

\section{Does Risk of Progression of NMIBC Matter for Outcome of Secondary MIBC?}

Risk of progression does convey adverse tumour biology; accordingly the outcome of RC for high-risk NMIBC is still compromised in some cases despite radical tumour removal. Accordingly, risk of progression at time of last TURBT for NMIBC prior to MIBC could reflect outcome. To date, only one series assessed risk of progression; de Vries et al. [19] stratified 54 NMIBC in one low/intermediate- and one high-risk group $(n=25$ and $n=29$, respec- 
tively), reporting 5 -year survival rates of 75 vs. $35 \%$, respectively. While numbers are comparably small in de Vries et al.'s and our series, we found comparable CSS rates for patients with high EORTC risk scores (67\% for the high-risk A group) and markedly advantageous outcome in patients with low EORTC risk scores of progression $(p=0.029)$.

\section{Which Conclusions Can Be Drawn for the \\ Management of High-Risk NMIBC?}

Outcome of secondary MIBC is generally critical; some $50 \%$ of the patients with cT2N0M0 had died from disease after 5 years. While this can be partly attributed to the high rate of upstaging to tumour stages $\geq \mathrm{pT} 3$ and/or $\mathrm{pN}+$, which is in accordance with the recent series by de Vries et al. and has been reported for up to $50 \%$ of all patients undergoing RC for MIBC $[19,22]$, it stresses the need for close follow-up of NMIBC at risk of progression. The present data furthermore demonstrate secondary MIBC subsequent to preceding NMIBC at high risk of progression to be brittle. Accordingly, debate of early RC should be considered previous to MIBC in high-risk NMIBC. This aspect is all the more challenging in the absence of reliable prognosticators of high-risk NMIBC. Recently, shortcomings of current grading systems for prognostic assessment were suggested [23]. Likewise, substaging of pT1 NMIBC in relation to depth of lamina propria invasion has been proposed due to inaccuracy of prognostic assessment $[24,25]$. Molecular markers seem not to suffice in this regard and require further assessment [5, 26, 27]. Thus, the present data emphasize the use of the EORTC risk score of progression in high-risk NMIBC to gain some additional prognostic information.

\section{Which Limitations of the Present Series Need to Be Taken into Account?}

The present series is marked by several limitations. For one, the present dataset is small, containing but 25 patients with secondary MIBC. To date, no considerably larger dataset which is fairly homogeneous and well-controlled for biasing factors has been reported. Second, while the present data were assessed prospectively, EORTC risk scores were assigned retrospectively for the earlier years of the present series based on clinicopathological characteristics assessed at the time of TURBT. Third, while control for use of BCG therapy had been exerted in secondary MIBC, no exact control for number of doses and duration of therapy could be achieved. Fourth, no patient in the present series obtained neoadjuvant chemotherapy despite more recent respective recommendations [3], which could compromise comparability to other data.

Outcome of Primary versus Secondary

MIBC after Radical Cystectomy

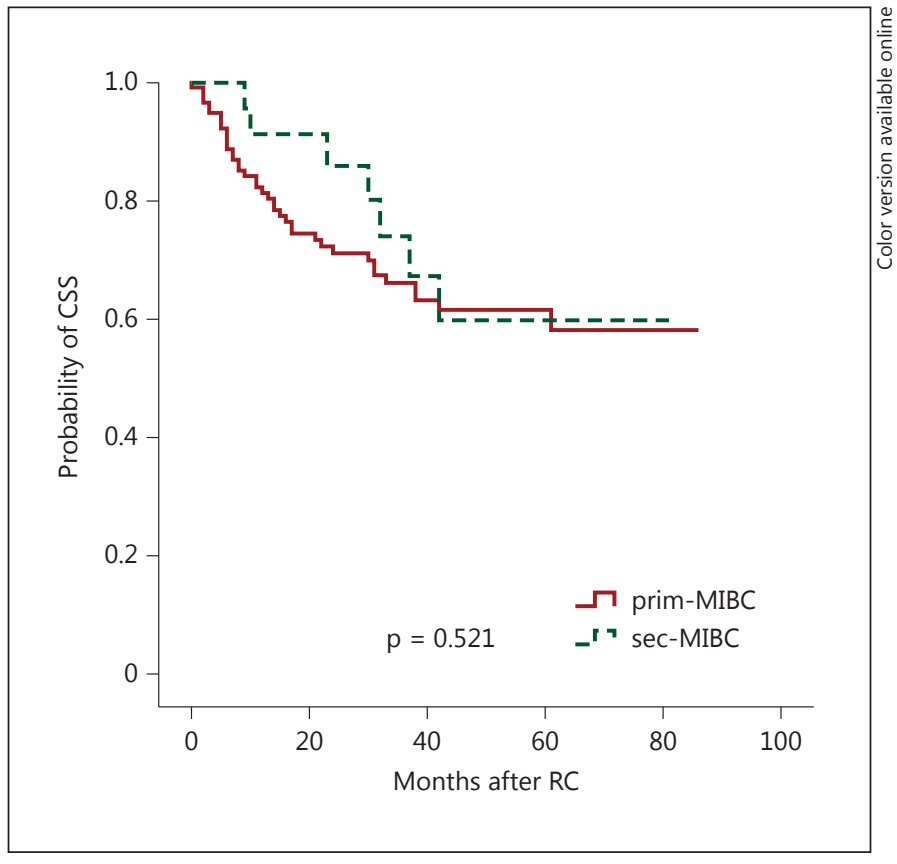

Fig. 2. Probability estimates of CSS in primary MIBC ( $\mathrm{n}=125$; prim-MIBC) and secondary MIBC $(n=25$; sec-MIBC).

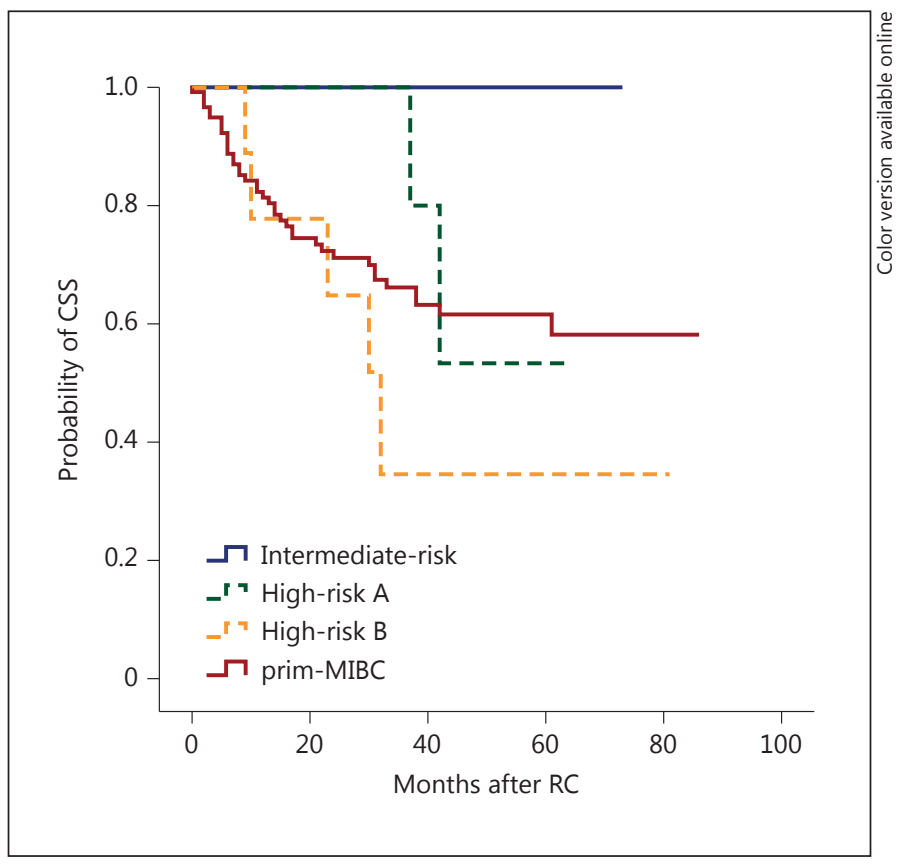

Fig. 3. Probability estimates of CSS after RC in primary MIBC ( $\mathrm{n}=125$; prim-MIBC) and secondary MIBC stratified according to the EORTC risk scores of progression predicting at time of last TURBT for NMIBC [intermediate-risk group (score 2-6) n $=6$ vs. high-risk A group (score 7-13) $\mathrm{n}=9$ vs. high-risk B group (score $14-23) \mathrm{n}=10]$. 


\section{Conclusions}

Outcome of secondary MIBC subsequent to previous NMIBC is not generally adverse compared to primary MIBC. In high-risk NMIBC debate of early RC should be considered, since high EORTC risk scores of progression at the last TURBT for NMIBC convey poor prognosis not only for progression to MIBC but also for outcome of secondary MIBC following RC.

\section{References}

$>_{1}$ Burger M, Catto JW, Dalbagni G, Grossman HB, Herr H, Karakiewicz P, Kassouf W, Kiemeney LA, La Vecchia C, Shariat S, Lotan Y: Epidemiology and risk factors of urothelial bladder cancer. Eur Urol 2013;63:234-241.

2 Babjuk M, Oosterlinck W, Sylvester R, Kaasinen E, Böhle A, Palou-Redorta J, Rouprêt M; European Association of Urology (EAU): EAU guidelines on non-muscle-invasive urothelial carcinoma of the bladder, the 2011 update. Eur Urol 2011;59:997-1008.

-3 Sylvester RJ, van der Meijden AP, Oosterlinck W, Witjes JA, Bouffioux C, Denis L, Newling DW, Kurth K: Predicting recurrence and progression in individual patients with stage $\mathrm{Ta}$ T1 bladder cancer using EORTC risk tables: a combined analysis of 2596 patients from seven EORTC trials. Eur Urol 2006;49:466-475; discussion 475-477.

4 Altieri VM, Castellucci R, Palumbo P, Verratti V, Sut M, Olivieri R, Manco R, Ricciardulli S, Nicolai M, Criniti P, Tenaglia RL: Recurrence and progression in non-muscleinvasive bladder cancer using EORTC risk tables. Urol Int 2012;89:61-66.

5 Gakis G, Schwentner C, Todenhoefer T, Stenzl A: Current status of molecular markers for prognostication and outcome in invasive bladder cancer. BJU Int 2012;110:233-237.

-6 Shapur N, Pode D, Katz R, Shapiro A, Yutkin V, Pizov G, Appelbaum L, Zorn KC, Duvdevani M, Landau EH, Gofrit ON: Predicting the risk of high-grade bladder cancer using noninvasive data. Urol Int 2011;87:319-324.

-7 Badalato GM, Gaya JM, Hruby G, Patel T, Kates M, Sadeghi N, Benson MC, McKiernan JM: Immediate radical cystectomy vs conservative management for high grade cT1 bladder cancer: is there a survival difference? BJU Int 2012;110:1471-1477.

$>8$ Jäger W, Thomas C, Haag S, Hampel C, Salzer A, Thüroff JW, Wiesner C: Early vs delayed radical cystectomy for 'high-risk' carcinoma not invading bladder muscle: delay of cystectomy reduces cancer-specific survival. BJU Int 2011;108:E284-E288.

$\checkmark 9$ Segal R, Yafi FA, Brimo F, Tanguay S, Aprikian A, Kassouf W: Prognostic factors and outcome in patients with T1 high-grade bladder cancer: can we identify patients for early cystectomy? BJU Int 2012;109:1026-1030.
10 Denzinger S, Fritsche HM, Otto W, Blana A, Wieland WF, Burger M: Early versus deferred cystectomy for initial high-risk pT1G3 urothelial carcinoma of the bladder: do risk factors define feasibility of bladder-sparing approach? Eur Urol 2008;53:146-152.

$>11$ Matsumoto K, Kikuchi E, Shirakawa H, Hayakawa N, Tanaka N, Ninomiya A, Miyajima A, Nakamura S, Oya M: Risk of subsequent tumour recurrence and stage progression in bacille Calmette-Guérin relapsing non-muscle-invasive bladder cancer. BJU Int 2012; 110:E508-E513.

$\$ 12$ Lee CT, Madii R, Daignault S, Dunn RL, Zhang Y, Montie JE, Wood DP Jr: Cystectomy delay more than 3 months from initial bladder cancer diagnosis results in decreased disease specific and overall survival. J Urol 2006; 175:1262-1267; discussion 1267.

13 Fahmy NM, Mahmud S, Aprikian AG: Delay in the surgical treatment of bladder cancer and survival: systematic review of the literature. Eur Urol 2006;50:1176-1182.

14 May M, Nitzke T, Helke C, Vogler H, Hoschke B: Significance of the time period between diagnosis of muscle invasion and radical cystectomy with regard to the prognosis of transitional cell carcinoma of the urothelium in the bladder. Scand J Urol Nephrol 2004;38: 231-235.

15 Greene FL, Gospodarowicz M, Wittekind C, et al: American Joint Committee on Cancer (AJCC) Staging Manual, ed 7. Philadelphia, Springer, 2009.

16 Sauter G, Algaba F, Amin M, et al: Tumours of the urinary system: non-invasive urothelial neoplasias; in Eble JN, Sauter G, Epstein Jl, Sesterhenn I (eds): WHO Classification of Tumors of the Urinary System and Male Genital Organs. Lyon, IARCC Press, 2004, pp 29-34.

17 Yiou R, Patard JJ, Benhard H, Abbou CC, Chopin DK: Outcome of radical cystectomy for bladder cancer according to the disease type at presentation. BJU Int 2002;89:374378.

18 May M, Helke C, Nitzke T, Vogler H, Hoschke B: Survival rates after radical cystectomy according to tumour stage of bladder carcinoma at first presentation. Urol Int 2004;72: 103-111.

$>19$ de Vries RR, Nieuwenhuijzen JA, Vincent A, van Tinteren $\mathrm{H}$, Horenblas S: Survival after cystectomy for invasive bladder cancer. Eur J Surg Oncol 2010;36:292-297.
20 Kotb AF, Kovac E, Kassouf W, Chin J, Fradet Y, Izawa J, Estey E, Fairey A, Rendon R, Cagiannos I, Lacombe L, Lattouf JB, Bell D, Drachenberg D, Aprikian AG: Radical cystectomy for clinically muscle invasive bladder cancer: does prior non-invasive disease affect clinical outcomes? World J Urol 2012;30:761-767.

21 Lee CT, Dunn RL, Ingold C, Montie JE, Wood DP Jr: Early-stage bladder cancer surveillance does not improve survival if high-risk patients are permitted to progress to muscle invasion. Urology 2007;69:1068-1072.

-22 Turker P, Bostrom PJ, Wroclawski ML, van Rhijn B, Kortekangas H, Kuk C, Mirtti T, Fleshner NE, Jewett MA, Finelli A, Kwast TV, Evans A, Sweet J, Laato M, Zlotta AR: Upstaging of urothelial cancer at the time of radical cystectomy: factors associated with upstaging and its effect on outcome. BJU Int 2012;110:804-811.

-23 Otto W, Denzinger S, Fritsche HM, Burger M, Wieland WF, Hofstädter F, Hartmann A, Bertz S: The WHO classification of 1973 is more suitable than the WHO classification of 2004 for predicting survival in pT1 urothelial bladder cancer. BJU Int 2011;107:404-408.

- 24 van Rhijn BW, Liu L, Vis AN, Bostrom PJ, Zuiverloon TC, Fleshner NE, van der Aa MN, Alkhateeb SS, Bangma $\mathrm{CH}$, Jewett MA, Zwarthoff EC, Bapat B, van der Kwast TH, Zlotta AR: Prognostic value of molecular markers, sub-stage and European Organisation for the Research and Treatment of Cancer risk scores in primary $\mathrm{T} 1$ bladder cancer. BJU Int 2012;110:1169-1176.

25 Bertz S, Denzinger S, Otto W, Wieland WF, Stoehr R, Hofstaedter F, Hartmann A: Substaging by estimating the size of invasive tumour can improve risk stratification in $\mathrm{pT} 1$ urothelial bladder cancer - evaluation of a large hospital-based single-centre series. Histopathology 2011;59:722-732.

26 Bertz S, Otto W, Denzinger S, Wieland WF, Burger M, Stöhr R, Link S, Hofstädter F, Hartmann A: Combination of CK20 and Ki-67 immunostaining analysis predicts recurrence, progression, and cancer-specific survival in pT1 urothelial bladder cancer. Eur Urol 2012 , Epub ahead of print.

27 Rink M, Cha EK, Green D, Hansen J, Robinson BD, Lotan Y, Sagalowsky AI, Chun FK, Karakiewicz PI, Fisch M, Scherr DS, Shariat SF: Biomolecular predictors of urothelial cancer behavior and treatment outcomes. Curr Urol Rep 2012;13:122-135. 